\title{
El Comercio Electrónico: ANTECEdentes, Definiciones Y SUJETOS
}

\author{
Cristian Alberto Robleto Arana \\ Profesor Adjunto de Derecho Civil y Mercantil \\ Universidad Centroamericana
}

$\mathrm{E}$

n esta oportunidad, presentaremos a nuestros lectores, la primera parte de uno de los temas más actuales en el Derecho Mercantil, titulado el "Comercio Electrónico". Tema que ha sido objeto de innumerables foros internacionales organizados por prestigiosas instituciones mundiales. Posteriormente, en las próximas ediciones de la revista jurídica estaremos ampliando el tema.

El presente artículo tiene como propósito conocer los antecedentes del comercio electrónico, citar definiciones de autores expertos en la tematica y relacionar los conceptos más utilizados en la práctica de la disciplina objeto de estudio.

La difusión de las tecnologías ligadas a internet junto a la nueva economía digital, ofrece a las empresas un importante número de oportunidades para hacerse más eficiente en términos de comunicación, visibilidad así como en la ampliación hacia nuevos mercados. Está comúnmente aceptado que comercio electrónico se ha convertido en una herramienta diaria de trabajo. Cada empresa debe considerar en qué forma utilizar los nuevos sistemas para incrementar su competitividad. No existen fórmulas mágicas o soluciones milagrosas, pero si que se presentan extraordinarias oportunidades de revalorizar los activos de la empresa.

Para maximizar los alicientes ofrecidos por el comercio electrónico las empresas necesitan integrar las nuevas tecnologías en su propia capacidad operacional, entendiendo como tal el desarrollo cotidiano de las herramientas precisas de trabajo. El escenario empresarial ha evolucionado, y el empresario debe entender que en la actualidad Internet ha pasado de constituir una oportunidad a ser una necesidad para la compañía.

\section{Antecedentes del comercio electrónico}

El desarrollo del comercio electrónico se da en cinco etapas ${ }^{1}$ :

Existen diversas teorías sobre los antecedentes del comercio electrónica, está es una de las que más se acerca a la verdad. 


\subsection{Primera etapa}

A partir de principios de los años sesenta, diversas universidades y el gobierno de Estados Unidos, buscaban la forma de crear una red que transportara información digitalizada y fraccionada desde un punto a otro, a través de un sistema descentralizado. Hacia fines de los años sesenta se puso en funcionamiento ARPANET, una red que unía a cuatro universidades dentro de Estados Unidos. Con el paso del tiempo se fueron integrando a dicha red nuevas instituciones y paralelamente se fueron desarrollando aplicaciones específicas con el fin de optimizar su uso. De esta manera, en 1972 se presenta por primera vez el e-mail (correo electrónico). Como culminación de la etapa se logra la estandarización de formatos y protocolos destinados a la interconexión de diversas redes.

\subsection{Segunda etapa}

Esta etapa se caracteriza por la ausencia de herramientas de trabajo efectivas. Si bien existían numerosas aplicaciones, no había un software confiable y estandarizado. Fuera del ámbito gubernamental o académico se encontraban pocos medios para interactuar comercialmente en la red, los sistemas de seguridad eran simples y la infraestructura de acceso a la red estaba poco desarrollada. Por estos motivos, grandes y pequeñas empresas se encontraban en una relativa igualdad de condiciones, por lo que esta etapa pueda ser caracterizada como etapa "democrática". Al no existir herramientas para el desarrollo de páginas o de motores de búsqueda. Cuando se realizaba una búsqueda activa sobre un tema determinado, pequeñas y grandes empresas aparecen en un mismo plano de participación. Así, la voluntad para actuar en nuevos campos y el ingenio pasaban a ser los requisitos esenciales para incursionar en el ámbito de la red.

En esta etapa, el uso de Internet no era visto como un recurso comercial atractivo. El modo de operar estaba dado por la transferencia que las empresas hacían de su material disponible a la red. Eventualmente algunas de ellas ofertaban sus productos, pero para realizar cualquier operación comercial se debía acudir a un lugar físico o bien hacer uso del teléfono, fax u otro medio de comunicación.

El alcance de Internet se limitaba, principalmente, a instituciones educativas y gubernamentales. Secundariamente, algunos proveedores de servicios de acceso a la red contaban con suscriptores (personas o empresas) que la utilizaban para la publicación de determinada información o como medio de comunicación mediante la utilización de servicios como el e-mail.

Las limitaciones del medio para ser utilizado como un vehículo eficaz del comercio, se hacían presentes con claridad. 


\subsection{Tercera etapa}

Casi a mediados de los años noventa, ya dentro de una nueva etapa, tanto el hardware como el software alcanzan un mayor grado de desarrollo, haciendo posible la utilización de interfases gráficas y ofreciendo mayor seguridad y velocidad. Esto permitió, por primera vez, una interacción real y fluida a través de la red.

Los motores de búsqueda se perfeccionaron y existe una mayor oferta de servicios on-line (America online, Compuserve, por mencionar algunos) que posibilitan que el público masivo comience a acercarse a Internet. La red deja de ser patrimonio exclusivo de científicos, gobiernos o instituciones educativas, abriéndose al público en general y a las empresas que comienzan a hacer uso de este medio publicando, viendo y comparando información.

De todas maneras, más allá de los logros que se experimentaban, Internet no dejaba de ser utilizada solamente como canal mediático adicional. No se verificaba comercio real puesto que no se percibía, dadas las dudas acerca de la seguridad en la transmisión de información, la utilidad y rentabilidad del medio.

Para comenzar a dar respuesta a esta problemática hay que abordar una nueva etapa.

\subsection{Cuarta etapa}

Esta nueva etapa se hace presente a partir de 1995 (la fecha podría resultar arbitraria) momento a partir del cual comienza el crecimiento "exponencial' de la red. Se produce un aumento de la infraestructura disponible, contándose con mayor cantidad de recursos y mayor velocidad de acceso, a lo que hay que agregar las ventajas dadas por el software que permiten sumar sonido y movimiento, mejorando simultáneamente la seguridad de las transacciones. En este período comienzan a utilizar agresivamente la red empresas que hoy son emblemas del comercio electrónico tales como yahoo (inicialmente motor de búsqueda) y Amazon.com (inicialmente venta de libros), Dello o Cisco [OCDE (1999)].

Durante 1997, INTER-NIC (compañía responsable de registrar las direcciones de los sitios en la red) reportó que se incorporaban nuevos sitios a un promedio de uno por minuto. Librerías, publicistas, firmas de servicios financieros, compradores y vendedores de todo tipo estaban inundando el espacio con sus archivos de información.

En esta etapa el comercio electrónico se desarrolla, principalmente, a través de tres vertientes que incluyen a: 
$\Rightarrow$ Empresas que ya mantenían lazos comerciales y que deciden utilizar la red para realizar sus operaciones comerciales y hacer uso de los medios tecnológicos disponibles para mejorar la eficiencia y fluidez de una relación comercial preexistente.

$\Rightarrow$ Empresas y personas innovadoras que comienzan a realizar operaciones comerciales percibidas como no "riesgosas".

$\Rightarrow$ Empresas y personas que utilizan la red para obtener información sobre bienes y servicios ofrecidos y, eventualmente, para iniciar una operación comercial.

\subsection{Quinta etapa}

La adopción definitiva del medio marca la etapa final en la cual ya no existen mayores problemas de capacidad, velocidad o seguridad (a pesar de no ser percibido este hecho por el público masivo). Internet, Intranet o Extranet son usadas, según convenga, para interconectar proveedores, distribuidores y socios. El público en general utiliza el medio con regularidad. En lo que respecta al uso de Internet como herramienta de comercio, ya no se encuentra con facilidad el antiguo espíritu "democrático". De alguna manera, la mayor o menor disponibilidad de recursos condiciona la participación de la empresa en el comercio electrónico. Para crear una Extranet que conecte empresas como las automotrices, las grandes cadenas de supermercados o de venta minorista con sus miles de proveedores, e hace necesario dedicar -a tales efectos- una gran cantidad de recursos (tiempo, dinero, recursos humanos).

Así, son diversas las maneras de poder acceder al comercio electrónico. Existen formas extremadamente sencillas como, por ejemplo, ofrecer bienes y servicios a través de empresas preestablecidas como Yahoo!, donde por unos US\$150 aproximadamente se puede ocupar un lugar en los shoppings virtuales o, a través de la creación de páginas web propias. Si bien esta última opción otorga mayor libertad e independencia, puede resultar extremadamente costosa, no sólo en lo relativo a su diseño sino también en lo concerniente a su mantenimiento y administración cotidiana.

En esta etapa son principalmente las grandes empresas las que, al decidir recuperar el espacio perdido, hacen su irrupción en el medio invirtiendo grandes sumas de dinero a través de canales publicitarios tradicionales e Internet. Empresas como Amazon.com o CDNOW.com, por ejemplo, lograron situarse en lo más alto del podio en términos de ventas dentro de sus categorías. Ahora deben enfrentar la competencia de empresas con una mayor trayectoria en el campo del comercio tradicional. En EE.UU., cadenas de librerías (como Barnes \& Noble) 
no sólo cuentan con sus tradicionales locales sino que, además, disponen de un servicio de ventas en Internet.

Otro ejemplo del uso de medios publicitarios masivos con el fin de atraer público hacia la red ha sido dado por la marca inglesa de ropa femenina Victoria's Secret que invirtió varios millones de dólares promocionando un desfile de moda transmitido en vivo a través de su página en Internet. Como resultado, diversos servidores de la red colapsaron debido a la congestión provocada por la gran cantidad de usuarios que buscaban acceder a la página de la marca [Napoli (1999)].

Esto ha demostrado que sí es posible una combinación efectiva de medios de publicidad tradicionales y el comercio electrónico y que la red ya no es un ámbito totalmente democrático como lo fue en sus inicios.

Ahora, a mayores recursos invertidos, mayor exposición y, normalmente, mayores ventas. De todos modos, lo verdaderamente alentador es que la red continúa manteniendo sus características esenciales de accesibilidad e interconectividad. Cualquier empresa o persona puede, potencialmente, acceder a ella y contar con un espacio donde ofrecer sus bienes y servicios. La capacidad de poseer una página en la red, participando así de las ventajas del comercio electrónico, es básicamente la misma para una empresa cuya facturación se mide en millones de dólares que para una empresa que inicia sus operaciones y cuenta con un presupuesto limitado.

Frente a la temática y problemática que envuelve el desarrollo del comercio electrónico y todo su potencial, tanto a nivel personal como empresarial y gubernamental se verifican distintas percepciones.

$\Rightarrow$ Hay quienes creen que el comercio electrónico es inaccesible por su complejidad o que su sector o industrial no puede beneficiarse si decidieran utilizarlo. De este modo, resulta más apropiado dejar el tema en manos de expertos. Esto no implica que desconozcan por completo la existencia del comercio electrónico. Simplemente se limitan, por ejemplo, a mantener un acceso a la red para navegar o una dirección de e-mail con el fin de utilizarlo como medio barato de comunicación.

$\Rightarrow$ Otros perciben la necesidad de tener cierta presencia en la red mas no ponderan la importancia de la interactividad permitida y requerida en el comercio electrónico. Se limitan a publicar "brochures" u otros materiales informativos pero no buscan, ni esperan una relación comercial a través de la red, ya que para ello prefieren utilizar los medios tradicionales como las visitas personales el uso del teléfono u otros medios de comunicación.

$\Rightarrow$ El verdadero comercio electrónico, aunque en forma rudimentaria, empieza para aquellos que se sitúen en la tercera etapa. Por un lado, la oferta me- 
diática es mucho más atractiva que en las etapas anteriores. Mejora tanto la calidad gráfica de las páginas como la facilidad para interactuar con ellas.

De todos modos, aún cuando realizan operaciones de comercio electrónico, los usuarios aquí situados todavía no confían ni en la fiabilidad ni en la seguridad del medio de modo que el comercio electrónico es utilizado para las tareas consideradas no estratégicas.

$\Rightarrow$ Quienes han entendido la importancia del comercio electrónico en su totalidad se situarán en la última etapa. Ya diseñan sus operaciones teniendo al comercio electrónico como objetivo inmediato. Sus sistemas están preparados para interactuar en el medio tomando las debidas precauciones en lo concerniente a fiabilidad y seguridad de la operación.

$\Rightarrow$ Finalmente, más allá de la posición tomada en relación a los avances tecnológicos, lo que vale es tomar conciencia de la necesidad que cada uno tiene de abordarlos. Los cambios tecnológicos implican, entre otras cosas, cumplir nuevos roles y enfrentar desafíos. En saber cómo responder a ellos radica la clave del éxito ${ }^{2}$.

\section{Definiciones de comercio electrónico}

Muchos son los estudiosos que han tratado de definir esta nueva modalidad mercantil, pero aún no se tiene una definición precisa sobre el tema, por ser de rápida evolución comercial. Para comprender el alcance de este término, es conveniente conocer algunas definiciones de expertos en la materia.

Según, el reporte preparado para Industria Canadá, 1998, por Davis, C. Hajnal, D. DeMatteis y M. Henderson, Requerimientos de Administración de Recursos para Comercio ${ }^{3}$, establece que el comercio electrónico incorpora "todas las transacciones de valor que involucren la transferencia de información, productos, servicios o pagos por medio de redes electrónicas" (ITAC, Noviembre 1997). El comercio electrónico "se refiere generalmente a todas las formas de transacciones relacionadas con las actividades comerciales, incluyendo organizaciones e individuos, que están basadas en el proceso y transmisión de datos digitalizados, incluyendo texto, sonido e imagen” (OECD 1997). Comercio electrónico "trata sobre hacer negocios electrónicamente". Está basado en el proceso y transmisión de datos electrónicos, incluyendo texto, sonido y video. Esto involucra muchas actividades diversas, incluyendo negociación electróni-

2 Gariboldi, Garardo. Comercio Electrónico: Conceptos y reflexiones básicas. Documento de divulgación. Buenos Aires. República de Argentina. Julio 1999.

3 Davis, C, Hajnal, C, DeMatteis, D. y Henderson, M, Requerimientos de Administración de Recursos para Comercio Electrónico (reporte preparado para Industria Canadá, 1998). http://www.COMERCIO ELECTRÓNICO $q$ que-escom-elec.html 
ca de bienes y servicios, envío de componentes digitales en línea, transferencia electrónica de fondos, negociación de segmentos electrónicos, conocimientos de embarque electrónicos, acciones comerciales, diseño e ingeniería cooperativa, fuentes en línea, compras, mercadeo directo al consumidor y servicio de post-venta. Esto involucra ambos productos (por ejemplo, artículos de consumo, equipo médico especializado) y servicios (por ejemplo, servicios de información, servicios financieros y legales); actividades tradicionales (por ej.: cuidado de la salud, educación) y nuevas actividades (por ej.: centros comerciales virtuales)" (Comisión Europea, 1997). El comercio electrónico, es un término genérico para describir la manera en que las organizaciones negocian electrónicamente. Usa un grupo de tecnologías para comunicarse con clientes u otras compañías, para llevar a cabo investigación o búsqueda de información o para conducir transacciones mercantiles. En todo caso el Internet es el más conocido de ellos, otros incluyen intranets, intercambio electrónico de datos (EDI) y tarjetas inteligentes. KPMG (1997).

Una definición abstracta de comercio electrónico que abarca la creciente virtualización de los tres componentes de un mercado (agentes, productos y procesos) ha sido llevada adelante por Whinston, Stahl y Choi (1997). Agentes, productos o procesos pueden ser físicos o virtuales. Las combinaciones posibles de agentes físicos o virtuales, productos y procesos, permiten la identificación de áreas de comercio tradicional, así como áreas principales donde "todos los tipos de servicios y procesos comerciales tienen el potencial de convertirse en productos digitales intercambiados en una red digital".

Según el estudio de situaciones del comercio electrónico en España de 19994, definen el comercio electrónico en sentido amplio, como cualquier forma de transacción o intercambio de información comercial basada en la transmisión de datos sobre redes de comunicación como Internet. En este sentido, el concepto de comercio electrónico no sólo incluye la compra y venta electrónica de bienes, información o servicios, sino también el uso de la Red para actividades anteriores o posteriores a la venta, como son:

$\Rightarrow$ la publicidad

$\Rightarrow$ la búsqueda de información sobre productos, proveedores, etc.

$\Rightarrow$ la negociación entre comprador y vendedor sobre el precio, condiciones de entrega, etc.

$\Rightarrow \quad$ la atención al cliente antes y después de la venta

$\Rightarrow$ trámites administrativos relacionados con la actividad comercial

4 Estudios de situaciones del Comercio Electrónico en España. Iniciativa de promoción e Identificación de Servicios Emergentes de Telecomunicaciones Avanzadas. (PISTA). Mayo 1999. http://sgc.mfom.es/set/ce 
$\Rightarrow$ la colaboración entre empresas con negocios comunes (a largo plazo o sólo de forma coyuntural)

En sentido estricto, se entiende como comercio electrónico, a las transacciones comerciales electrónicas, es decir, de compraventa de bienes o prestaciones de servicios, así como las actividades y negociaciones previas y otras actividades ulteriormente relacionadas con las mismas, aunque no sean estrictamente contractuales. Ejemplo: pagos electrónicos, a través de correo electrónico o WWWW- World Wide Web ${ }^{5}$, ambas aplicaciones de Internet o IED -Intercambio electrónico de datos.

De tal forma que la definición en sentido amplio debe denominarse como intercambio o transmisión electrónica de datos, en cambio la segunda definición en sentido estricto debe considerarse como comercio electrónico. Por lo tanto, la tradicional forma de contratar es sustituida por la novedosa forma electrónica.

La definición en sentido estricto abarca otras actividades, dos tipos de actividades que engloba el comercio electrónico, estas son: el pedido electrónico de bienes materiales que se entrega a través de los canales tradicionales como el correo o a través de los servicios de mensajería, ésta es denominado como comercio electrónico indirecto, puesto que depende de factores externos, como el sistema de transporte; en segundo lugar, tenemos el pedido, el pago y la entrega en línea de bienes y servicios intangibles, como programas informáticos, revistas electrónicas, servicios recreativos y de información, es denominado como comercio electrónico directo, aprovechando todo el potencial del mercando electrónico.

Moira Jacobs, responsable de la oficina del Departamento de Comercio del gobierno de los Estados Unidos, la cual se encarga de proporcionar servicios integrales de consultoría en materia de Internet y Comercio Electrónico a través de más de 100 centros, a micro, pequeñas y medianas empresas estadounidenses interesadas en exportar en línea sus mercancías y servicios, define el Comercio Electrónico como: "Cualquier transacción entre compradores y vendedores a través de medios electrónicos". ${ }^{6}$

Vadim Levitin, consultor de la compañía ECRX.COM, refiere la siguiente definición de Comercio Electrónico, la cual procede de los reconocidos consultores de The Gartner Group: "El Comercio Electrónico es un envolvente conjunto de herra-

\footnotetext{
Actualmente, el WWW y el correo electrónico, ambos aplicación de Internet, juegan un papel importante en el comercio electrónico, como instrumento de difusión de información comercial a potenciales compradores de bienes o servicios, pero también como medio de realización de una transacción electrónica efectiva, de forma prácticamente interactiva y on-line (caso del Web), o por medio de procedimiento de almacenamiento y reenvío de un mensaje a través de los denominados agentes de transferencias de mensajes (caso del correo electrónico).

6 Moira Jacobs: "International E-Business Oportunities and Challenges: A Small Business Perspective". Ponencia presentada en The 1999 e-Business Opportunities, Challenges, and Winning Strategies. Universidad de Montana en Missoula y The Montana World Trade Center, Estados Unidos, 2-5 de agosto de 1999.
} 
mientas de tecnologías de información, así como estrategias de negocios destinadas a favorecer la realización de prácticas comerciales de forma electrónica". 7

Gregory Martín, presidente de CORRILLIAN, firma dedicada a proporcionar servicios de consultoría estratégica en materia de Internet, considera que el Comercio Electrónico es: "es emergente ambiente de negocios derivado de la convergencia de las computadoras y las comunicaciones".

El comercio electrónico es la venta a distancia ${ }^{9}$ que aprovechando las grandes ventajas que proporcionan las nuevas tecnologías de la información, como la ampliación de la oferta, la interactividad y la inmediatez de la compra, con la particularidad que se puede comprar y vender a quién se quiera, y, dónde y cuándo se quiera. Es toda forma de transacción comercial o intercambio de información, mediante el uso de Nueva Tecnología de Comunicación entre empresas, consumidores y administración pública.

El estudio realizado por la OMPI ${ }^{10}$, en Mayo del 2000. Define conceptos "comercio" y "electrónico", por separado.

Electrónico. Por "electrónico" cabe entender la infraestructura mundial de tecnologías y redes de la informática y las telecomunicaciones que permiten el procesamiento y la transmisión de datos digitalizados. Numerosos estudios han abor-

7 Vadim, Levtin: "Getting Started in Cyperspace: Reviewing the Basics". Ponencia presentada en The 1999 eBusiness Conference. Universidad de Montana en Missoula y The Montana World Trade Center, Estados Unidos, 2-5 de agosto de 1999.

8 Martin,Greg. "Internet Banking in Exploding e-Space. Customer Compliant or Customer Complacent". Ponencia presentada en The 1999 e-Business Conference, Universidad de Montana en Missoula y The Montana World Trade Center, Estados Unidos, $2-5$ de agosto de 1999.)

9 El Diario Oficial de las Comunidades Europeas del cuatro de junio de 1997 publicó la Directiva 97/7/CE relativa a la protección de los consumidores en materia de contratos a distancia. El tema ofrece un gran interés en la actualidad, ya que afecta a las operaciones de comercio electrónico que de manera progresiva van realizándose a través de Internet.

Si hasta ahora podía ponerse en duda el carácter de venta a distancia de una transacción efectuada a través del correo electrónico, a partir de la transposición de esta Directiva, los contratos celebrados mediante este tipo de comunicaciones entrarán de lleno en el régimen establecido por la misma.

Las principales repercusiones que se derivan de la aplicación de la normativa citada se resumen a continuación:

$\Rightarrow$ El consumidor dispondrá de un plazo mínimo de siete días laborables, a partir de la recepción del producto, para rescindir el contrato sin penalización alguna, y sin indicación de los motivos. El único gasto que podría imputarse al consumidor es el coste directo de la devolución de las mercancías al proveedor.

$\Rightarrow$ Quedarán exceptuados del derecho de arrepentimiento los productos que puedan ser reproducidos fácilmente: grabaciones sonoras o de vídeo, programas informáticos, publicaciones periódicas, etc.

$\Rightarrow$ Salvo pacto en contrario, el proveedor deberá suministrar el pedido en el plazo máximo de treinta días a partir del día siguiente a aquél en que el consumidor le haya comunicado su pedido.

$\Rightarrow$ El consumidor podrá solicitar la anulación de un pago en caso de utilización fraudulenta de su tarjeta de pago en el marco de contratos a distancia.

$\Rightarrow$ En caso de utilización fraudulenta, se restituirán las sumas abonadas en concepto de pago.

$\Rightarrow$ Se prohíbe el suministro de bienes o servicios que no hayan sido solicitados previamente por el consumidor, cuando dichos suministros incluyan una petición de pago. La falta de respuesta en tales situaciones no podrá considerarse como consentimiento.

$\Rightarrow$ Los servicios financieros quedan excluidos del ámbito de la Directiva.

10 OMPI- Estudio sobre Comercio Electrónico y Propiedad Intelectual. Mayo-2000 
dado la evolución desde las primeras redes privadas, en las que las transacciones electrónicas han sido moneda corriente durante varias décadas, hasta las redes abiertas con protocolos de uso público, como Internet. Un rasgo común de esas redes es que operan sobre la base de propósitos definidos de manera específica y están destinadas exclusivamente para los participantes autorizados.

En cambio, Internet permite que un número potencialmente ilimitado de participantes que quizá no hayan tenido contacto previo se comuniquen y realicen transacciones en una "red abierta" ${ }^{11}$ ", que no exige dispositivos de seguridad. Internet ha evolucionado rápidamente, de una red científica y académica, a una red cuyo principal elemento distintivo, la World Wide Web, ha sido adoptado a gran escala $^{12}$. El carácter abierto de esta red, junto con su naturaleza multifuncional y un acceso cada vez más barato, han impulsado el potencial del comercio electrónico. Al mismo tiempo, la red abierta proporciona acceso a un medio digital en el que pueden hacerse y transmitirse con facilidad múltiples copias perfectas de textos, imágenes y sonidos, propiciando el uso indebido de marcas, lo que origina problemas para los titulares de derechos de propiedad intelectual.

Comercio. En este contexto, la palabra "comercio" hace referencia a una serie cada vez mayor de actividades que tienen lugar en redes abiertas -compra, venta, comercio, publicidad y transacciones de toda índole- que conducen a un intercambio de valor entre dos partes. Cabe citar como ejemplos las subastas, los servicios bancarios y demás servicios financieros, la venta de programas, y un número creciente de sitios Internet que ofrecen una amplia gama de bienes o servicios de consumo, todos ellos en línea. En lo que atañe a los consumidores,

11 El término "red abierta" hace referencia a una red, como Internet, que utiliza protocolos no privados creados mediante un proceso de creación de normas sin restricciones. Internet se basa en un protocolo abierto y no privado, denominado Protocolo de Control de Transmisión/Protocolo Internet (TCP/IP), y utiliza un sistema de codificación normalizado, el lenguaje de marcación hipertexto (HTML), para representar datos de manera gráfica en la World Wide Web. Véase "The Economic and Social Impacts of Electronic Commerce: Preliminary Findings and Research Agenda”, Organización de Cooperación y Desarrollo Económicos, Capítulo 2, pág. 1 (1999), en http://www.oecd.org/subject/e_commerce/summary.htm. La World Wide Web, desde una perspectiva técnica, hace referencia a los servidores de hipertexto (servidores HTTP), que son servidores que permiten combinar texto, gráficos y ficheros audio". Véase "Challenges to the Network: Internet for Development", Unión Internacional de Telecomunicaciones (UIT), Glosario (1999).

12 Internet empezó siendo un medio estrictamente no comercial. Para conseguir referencias de diversos documentos sobre los comienzos de Internet, véase "History of the Internet", sitio Web de Internet Society, en http://www. isoc.org/internet-history/. Véase también Townsend, nota 7, que explica que Internet se puso en marcha en los años 60 como un proyecto de investigación denominado ARPANET, llevado a cabo por el Departamento de Defensa del Gobierno de los Estados Unidos de América. Lanzada en 1990, la World Wide Web fue desarrollada por científicos del Laboratorio Europeo de Física de Partículas de Ginebra (CERN). A finales de 1993, el Centro Nacional para las aplicaciones de las supercomputadoras es de EE.UU. (NCSA) dio a conocer el primer navegador Internet integrado, Mosaic, cuya interfaz gráfica de usuario hacía más sencilla la navegación por Internet. Sin embargo, no fue hasta 1994 cuando Netscape Communications Corporation, fundada por el Dr. James Clark y Marc Andreessen, sacó a la luz y popularizó el primer navegador comercial de Internet utilizado a gran escala. Véase el sito Web de Netscape en http://home.netscape.com/company/about/ backgrounder.html\#market. 
un sitio Web comercial que hace algún tiempo tuvo gran éxito es el sitio de venta de libros, mediante el cual el consumidor puede encargar un libro (y pagar por medios electrónicos como la tarjeta de crédito) que le será enviado por correo a su domicilio ${ }^{13}$. En la actualidad es posible adquirir otros productos mediáticos, como la música, descargándolos directamente en forma digital en la computadora del consumidor (o en otros dispositivos digitales ${ }^{14}$ ).

La ley modelo de la CNUDMI ${ }^{15}$ sobre Comercio Electrónico -UNCITRAL-, no define el comercio electrónico, pero su artículo dos se refiere a los EDI intercambio electrónico de datos denominado. Conviene destacar que al redactar la ley modelo se tuvo presente las técnicas modernas de comunicación, tales como el EDI y el correo electrónico, los principios en las que se inspiran, así como sus disposiciones, son igualmente aplicables a otras técnicas de comunicación como el fax.

\section{Los objetivos de la ley modelo son siguientes:}

a) Ofrecer a los legisladores nacionales una serie de normas aceptadas en el ámbito internacional a fin de suprimir los obstáculos legales y darle al comercio electrónico una plataforma firme y legal.

b) Ayudar a los usuarios del comercio electrónico a superar los obstáculos legales que su utilización ofrece.

c) Buscar como darle una mayor eficacia al comercio electrónico internacional para la cual propone:

$\Rightarrow$ La utilización de técnicas de comunicación.

$\Rightarrow$ Trato igual a los usuarios de documentos en papel y a los usuarios de documentación informatizada.

$\Rightarrow$ Otorgar fuerza probatoria a los mensajes de datos siempre que s sean legibles e interpretables, es decir, obedezcan al concepto de accesible.

Actualmente el comercio electrónico es conocido como un mercado abierto, porque el comercio que se lleva a cabo es, en la mayor parte del tiempo sin acuerdos previos bilaterales, entre partes que no se conocen. Esta modalidad de comercio facilita las relaciones ocasionales o a corto plazo entre las partes

13 Véase Amazon.com, en http://www.amazon.com, que ha ampliado la variedad de la oferta de su sitio Web para competir con los grandes almacenes, y ha incluido libros, juguetes y juegos, computadoras y aparatos electrónicos, artículos deportivos, música y vídeos, ropa femenina y muchas otras cosas.

14 Véase, por ejemplo, "Quiet, Please, Test in Progress", International Herald Tribune (25 de octubre de 1999). En el artículo, se ofrece una visión global de una prueba/ensayo llevada a cabo de forma conjunta por International Business Machines (IBM) y varios sellos discográficos de primer orden para vender música directamente por Internet (mediante cables módem de alta velocidad) a cerca de 1000 hogares del área metropolitana de San Diego, California.

15 Comisión de las Naciones Unidas para el Derecho Mercantil Internacional-CNUDMI 
negóciales. Las transacciones comerciales, a través del comercio electrónico se limitaba a relaciones interempresariales a través de redes propias, hoy en día se está ampliando y convirtiendo en una compleja malla de actividades a nivel mundial entre participantes (empresas, particulares, conocidos y desconocidos) que utilizan redes abiertas de ámbito mundial y cuyo número no deja de crecer. Hoy en día aunque el nivel del comercio en Internet es todavía relativamente bajo, (en éste sentido se dice que actualmente es un universo de navegadores más que de compradores) son cada vez más habituales las compras en redes, y de cara a un futuro irá en aumento, incluyendo actividades comerciales de mayor envergadura ${ }^{16}$.

En ese caso, Internet actúa como un poderoso medio para mejorar la calidad de la gestión y los servicios, creando así nuevas relaciones entre consumidores y proveedores, o afianzando las ya existentes, y aportando al mismo tiempo más eficacia y transparencia a las operaciones. Es un potente mecanismo para reducir los costos de carácter general, en particular, los asociados a la producción, los inventarios, la gestión de ventas, la distribución y la compra.

Cabe mencionar, al menos dos características de este comercio que tiene lugar en redes digitales. En primer lugar, su naturaleza internacional. Los medios electrónicos descritos anteriormente han creado un medio mundial sin límites, de manera que ninguna empresa que ofrezca bienes o servicios en Internet tenga que dirigirse a un mercado geográfico concreto. La creación de un sitio Web comercial puede proporcionar, incluso a una empresa pequeña, acceso a mercados y a usuarios de Internet de todo el mundo. En segundo lugar, la naturaleza interdisciplinaria del comercio electrónico, y el consiguiente impacto en las fuerzas de convergencia. Tanto las empresas grandes como las pequeñas están comprobando que lo que diferenciaba hasta ahora a los sectores comerciales —en razón de las características físicas de los bienes o servicios y de los diversos medios empleados para su distribución (por ejemplo, libros, películas, discos compactos, televisión, radio y retransmisiones por la Web) — está perdiendo su razón de ser, y eso origina presiones competitivas para reestructurar las actividades dentro de las industrias y entre ellas, lo que supone nuevas oportunidades y a la vez problemas para las empresas ${ }^{17}$.

En conclusión, se entiende como comercio electrónico, una modalidad de compra no presencial o a distancia que consiste en la adquisición de bienes (por ej. un

${ }^{16}$ Martí, Andreu, María del Mar y otros. Curso sobre protección jurídica de los consumidores. 2000

${ }^{17}$ Para un debate general sobre los cambios que se están produciendo en el mercado como consecuencia del comercio electrónico, véase "The Economic and Social Impacts of Electronic Commerce: Preliminary Findings and Research Agenda", Organización de Cooperación y Desarrollo Económicos, Capítulo 5 (1999), en http:// www.oecd.org/subject/e_commerce/summary.htm. 
juguete, un libro, etc.) o de servicios (por ej. un billete de avión, acceder a algunos estudios universitarios, acceder a una base de datos actualizada sobre recetas de cocina, etc.) a través de la llamada "vía electrónica", es decir, a través de equipos electrónicos de tratamiento y almacenamiento de datos, y que se transmite, canaliza y recibe íntegramente a través de hilos, radio, medios ópticos o cualquier otro medio electromagnético.

Visto desde otro punto de vista empresarial, el comercio electrónico es un medio de hacer posible y soportar tales cambios a escala global. Permite a las empresas ser más eficientes y más flexibles en sus operaciones internas, trabajar más estrechamente con sus suministradores y dar mejor respuesta a las necesidades y expectativas de sus clientes. Les permite seleccionar los mejores proveedores, sin tener en cuenta su localización geográfica, y vender en un mercado global.

\section{Conceptos relacionados con el comercio electrónico}

\subsection{Mensaje de datos}

Se entenderá, la información generada, enviada, recibida o archivada o comunicada por medios electrónicos, ópticos o similares, como pudieran ser, entre otros el intercambio electrónico de datos (EDI), correo electrónico, el telegrama, el telex o telefax ${ }^{18}$.

También, se refiere a toda información consignada en soportes informáticos aunque no esté destinada a ser comunicada. Es posible que algunas legislaciones utilicen el concepto de "información consignada"

Cuando se refiere a los "medios similares", esto significa que la ley modelo no sólo fue destinada a los medios actuales, sino a cualquier otro medio que un futuro aparezcan.

El mensaje de datos, abarca todo tipo de mensajes generados, archivados o comunicados, en forma básicamente distinta del papel.

Se entiende que mensaje de datos, abarca el supuesto de revocación o modificación de un mensaje de datos, aunque se supone que el contenido de un mensaje de datos es invariable, pero ese mensaje puede ser revocado o modificado por otro mensaje de datos.

Para ley modelo de comercio electrónico, firmas electrónicas y mensajes de datos de Ecuador, del 27 de febrero del 2002, en sus disposiciones finales, establece que los mensajes de datos, es toda información, creada, generada, procesada, enviada, recibida, comunicada o archivadas por me-

18 Ley Modelo de la CNUDMI, Artículo: 2.a 
dios electrónicos que puede ser intercambiada por cualquier medio. Serán considerados como mensajes de datos, sin que esta enumeración límite su definición, los siguientes: documentos electrónicos, registros electrónicos, correo electrónico, servicios web, telegramas, telex, fax o intercambio electrónico de datos.

\subsection{Intercambio electrónico de datos}

Consiste en la transmisión electrónica de información de una computadora a otra, estando estructurada la información conforme a alguna norma técnica convenida al efecto ${ }^{19}$.

El recursos a los modernos medios de comunicación tales como correo electrónico y el intercambio electrónico de datos, se ha difundido en los negocios de las operaciones comerciales internacionales y cabe prever que el empleo de esas vías de comunicación sea cada vez mayor a medida que se vaya difundiendo el acceso a ciertos soportes técnicos como el Internet y otras grandes vías de comunicación transmitida en forma electrónica, no obstante la transmisión de datos da cierta trascendencia jurídica en forma de mensaje sin soporte de papel pudiera verse obstaculizada por ciertos impedimentos legales al empleo de mensajes electrónicos, o por la incertidumbre que pudiera haber sobre la validez o eficacia jurídica de éstos mensajes. La ley modelo tiene como objetivo ofrecer al legislador nacional un conjunto de reglas aceptables en el ámbito internacional que le permitan eliminar algunos de esos obstáculos jurídico, con miras a crear un marco jurídico que permita un desarrollo más seguro de las vías electrónicas de negociación, designada por el nombre de comercio electrónico ${ }^{20}$.

\subsection{Iniciador de un mensaje de datos}

Es toda persona que al tenor del mensaje haya actuado por su cuenta o en cuyo nombre se haya actuado para enviar o generar ese mensaje antes de ser archivado, si éste es el caso, pero que no haya actuado a título de intermediario con respecto a él ${ }^{21}$.

\subsection{Destinatario del mensaje de datos}

Es la persona designada por el iniciador, para recibir el mensaje, pero que no esté actuando a título de intermediario con respecto a él ${ }^{22}$.

\footnotetext{
Ley Modelo de la CNUDMI, Artículo: 2.b

${ }^{20}$ Guía para la incorporación del derecho interno de la ley modelo de la CNUDMI sobre Comercio Electrónico.

21 Ley Modelo de la CNUDMI. Artículo:2.c

22 Ley Modelo de la CNUDMI. Artículo:2.d
} 


\subsection{Intermediario}

Es toda persona que actúa por cuenta de otra, envíe, recibe o archive dicho mensaje o preste algún otro servicio con respecto a él ${ }^{23}$.

Para la ley modelo, las relaciones entre iniciador y destinatario de un mensaje de datos, no se negarán efectos jurídicos, validez o fuerza obligatoria a una manifestación de voluntad u otra declaración por la sola razón de haberse hecho en forma de mensaje de datos ${ }^{24}$.

Ejemplo: los vendedores al por mayor y al por menor proporcionan servicios esenciales en los mercado físicos.

\subsection{Sistema de información}

Es todo sistema utilizado para generar, enviar, recibir, archivar, procesar de alguna otra forma, mensajes de datos ${ }^{25}$.

Esta definición engloba toda la gama de los medios técnicos empleados para transmitir, recibir y archivar información. Por ejemplo: el sistema de información se refiere en algunos casos en una red de comunicación y en otros casos en un buzón electrónico o incluso en una telecopiadora ${ }^{26}$.

Para ley de Comercio electrónico, firmas electrónicas y mensajes de datos de Ecuador, del 27 de febrero del 2002, en sus disposiciones finales, establece que el sistema de información es todo dispositivo físico o lógico, utilizado para crear, generar, enviar, recibir, procesar, comunicar o almacenar, de cualquier forma, mensaje de datos.

La importancia jurídica del sistema de información radica principalmente es en cuanto a la fecha, hora de remisión del mensaje de datos, así como de su recepción por parte del destinatario.

\section{Sujetos que intervienen en el comercio electrónico}

Los participantes en el mercado electrónico no están limitados a las empresas, por el contrario, la era digital nos afecta a todos. Sus principales áreas de aplicación se encuentran:

\section{Entre empresas y consumidor (B2C), o mejor conocido como Business} to consumer). La expresión Business to consumer, designa el conjunto de las transacciones comerciales de bienes y servicios entre empresas y consumidores finales. El comercio electrónico entre empresas y consumidores ha

23 Ley Modelo de la CNUDMI. Artículo:2.e

${ }^{24}$ Ley Modelo de la CNUDMI. Artículo: 12

${ }^{25}$ Ley Modelo de la CNUDMI. Artículo: 2.f

${ }^{26}$ Guía para la incorporación al derecho interno de la ley modelo de la CNUDMI sobre comercio electrónico. 
evolucionado poco todavía en el ámbito global, de todas maneras se prevé, que constituirá un peso poco importante de las transacciones comerciales electrónicas. Más que sustitutivo, el comercio electrónico entre empresas y consumidores se presenta como complemento al tradicional, porque depende principalmente de la comodidad para el uso del cliente.

Entre empresa y empresa (B2B o mejor conocido como Business to Business). El mercado entre empresa y empresa comprende todas las transacciones comerciales efectuadas entre una empresa y otras organizaciones, sean éstas colaboradores comerciales, suministradores, clientes o instituciones. En este mercado suceden la mayor parte de las actividades de comercio electrónico realizadas en el mundo. Una primera tipología de comercio entre empresas nace de las relaciones fundadas en una sólida relación de colaboración ínter industrial, que a menudo ya se servían de sistemas de intercambio electrónico de datos. ${ }^{27}$ La evolución de las transacciones electrónicas entre empresas es lenta, aunque actualmente ocupa el 80\% del total de la actividad comercial electrónica.

Un ejemplo de la categoría empresa-empresa sería una compañía que usa una red para ordenar pedidos a proveedores, recibiendo los cargos y haciendo los pagos. Está establecida desde hace bastantes años, usando en particular Intercambio Electrónico de Datos (EDI, Electronic Data Interchange) sobre redes privadas o de valor añadido.

Entre consumidor y consumidor (C2C, o mejor conocido como Consumer to-consumer). Es la popular y reciente forma de contracambio y es más conocida con la denominación de subasta on-line y se da entre un consumidor y otro consumidor, es decir entre dos personas naturales.

Entre consumidor y administración ${ }^{28}$ (C2A, o mejor conocida como consumers to administrations) se da entre los ciudadanos y las administraciones públicas cuando éstos interactúan con ellas a efectos de realizar la presentación de las declaraciones juradas y/o el pago de los tributos, obtener asistencia informativa y otros servicios.

$\checkmark$ Entre empresa y administración ${ }^{29}$ (B2A, o mejor llamada Business to administrations). Es la relación que se da cuando las administraciones públicas

27 Articulo 2 Ley Modelo sobre Comercio Electrónico. Es la transferencia electrónica de información de ordenador a ordenador, utilizando un acuerdo estándar para estructurar una información.

${ }^{28}$ La categoría consumidor-administración, no acaba de emerger. Sin embargo, a la vez que crecen tanto las categorías empresa-consumidor y empresa-administración, los gobiernos podrán extender las interacciones electrónicas a áreas tales como los pagos de pensiones o el autoasesoramiento en devoluciones de tasas.

${ }^{29}$ La categoría empresa administración cubre todas las transacciones entre las empresas y las organizaciones gubernamentales. Por ejemplo, en USA las disposiciones gubernamentales se publicitan en Internet y las compañías pueden responder electrónicamente. Generalmente esta categoría está empezando, pero puede crecer rápidamente si los gobiernos la usan para sus operaciones para promover la calidad y el crecimiento del comercio electrónico. Además, las administraciones pueden ofrecer también la opción del intercambio electrónico para transacciones como determinados impuestos y el pago de tasas corporativas. 
actúan como agentes reguladores y promotores del comercio electrónico y como usuarias del mismo, interactuando para tal efecto con las empresas.

En conclusión en el comercio electrónico participan como actores principales las empresas, los consumidores y las administraciones públicas. Así se distinguen tres tipos básicos de comercio electrónico.

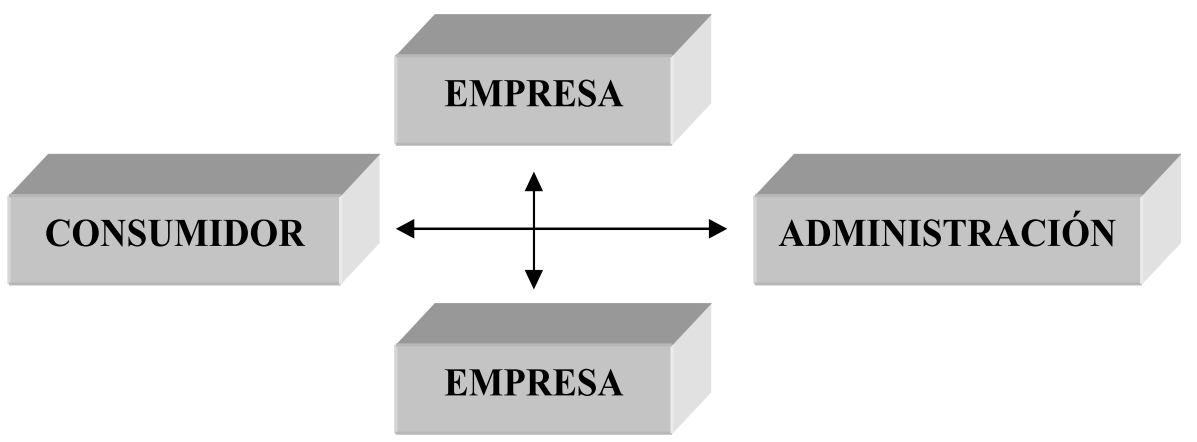

Las empresas intervienen como usuarias (vendedoras o compradoras) y como proveedores de herramientas o servicios de soporte para el comercio electrónico: proveedores de servicios de información de certificación de claves públicas, instituciones financieras, etc. Por su parte la administración pública actúa como agentes reguladores y promotores del comercio electrónico o como usuarias del mismo (por ejemplo en los procedimientos de contratación pública o de compra por la administración).

Los consumidores participarán en dos formas adicionales de comercio electrónico además del B2C, por una parte, el comercio electrónico directo entre consumidores (venta directa entre particulares) y, por otra, las transacciones entre ciudadanos y administración (pagos de prestaciones sociales, pago de impuestos, etc)

También, comprende el desarrollo de operaciones comerciales y de servicios de empresas con el gobierno (B2G); servicios del gobierno a consumidores y ciudadanos (G2C); transacciones de gobierno a gobierno (G2G)

\section{BIBLIOGRAFÍA}

- Estudios de situaciones del Comercio Electrónico en España. Iniciativa de promoción e Identificación de Servicios Emergentes de Telecomunicaciones Avanzadas. (PISTA). Mayo $1999 \mathrm{http} / / / \mathrm{sgc} . \mathrm{mfom} . \mathrm{es} / \mathrm{set} / \mathrm{ce}$ 
- Estudio de situación del comercio electrónico en España. Iniciativa de promoción e identificación de de servicios emergentes de telecomunicaciones avanzadas. (PISTA). http// www.sgc.mfom.es/sat/ce/indice.html

- Davis, C. Hajnal, C, DeMatteis, D. y Henderson, M, Requerimientos de Administración de Recursos para Comercio Electrónico (reporte preparado para Industria Canadá, 1998) http://www.COMERCIO ELECTRÓNICOlque-es-com-elec.html

- Diario Oficial de las Comunidades Europeas del cuatro de junio de 1997 publicó la Directiva 97/7/CE relativa a la protección de los consumidores en materia de contratos a distancia.

- Gariboldi, Garardo. Comercio Electrónico: Conceptos y reflexiones básicas. Documento de divulgación 4. Buenos Aires. República de Argentina. Julio 1999.

- Guía para la incorporación al derecho interno de la ley modelo de la CNUDMI sobre comercio electrónico.

- Martí, Andreu, y otros. Curso sobre protección jurídica de los consumidores. 2000

- Martin, Greg: "Internet Banking in Exploding e-Space: Customer Compliant or Customer Complacent". Ponencia presentada en The 1999 e-Business Conference, Universidad de Montana en Missoula y The Montana World Trade Center, Estados Unidos, 2-5 de agosto de 1999.

- Moira, Jacobs,: "International E-Business Oportunities and Challenges: A Small Business Perspective". Ponencia presentada en The 1999 e-Business Opportunities, Challenges, and Winning Strategies. Universidad de Montana en Missoula y The Montana World Trade Center, Estados Unidos, 2-5 de agosto de 1999.

- Levtin, Vadim: "Getting Started in Cyperspace: Reviewing the Basics". Ponencia presentada en The 1999 e-Business Conference. Universidad de Montana en Missoula y The Montana World Trade Center, Estados Unidos, 2-5 de agosto de 1999.)

- OMPI- Estudio sobre Comercio Electrónico y Propiedad Intelectual. Mayo-2000

- Ley Modelo de la CNUDMI- Comisión de las Naciones Unidades para el Derecho Mercantil Internacional. 